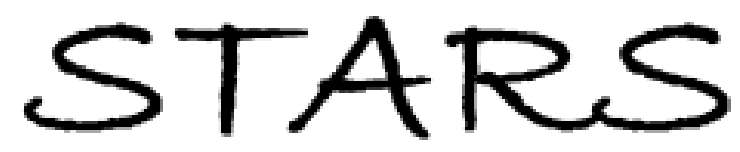

University of Central Florida

STARS

$1-1-2003$

\title{
Tunable Fresnel lens using nanoscale polymer-dispersed liquid crystals
}

\author{
Hongwen Ren \\ University of Central Florida \\ Yun-Hsing Fan \\ University of Central Florida \\ Shin-Tson Wu \\ University of Central Florida
}

Find similar works at: https://stars.library.ucf.edu/facultybib2000

University of Central Florida Libraries http://library.ucf.edu

This Article is brought to you for free and open access by the Faculty Bibliography at STARS. It has been accepted for inclusion in Faculty Bibliography 2000s by an authorized administrator of STARS. For more information, please contactSTARS@ucf.edu.

\section{Recommended Citation}

Ren, Hongwen; Fan, Yun-Hsing; and Wu, Shin-Tson, "Tunable Fresnel lens using nanoscale polymerdispersed liquid crystals" (2003). Faculty Bibliography 2000 s. 3983.

https://stars.library.ucf.edu/facultybib2000/3983

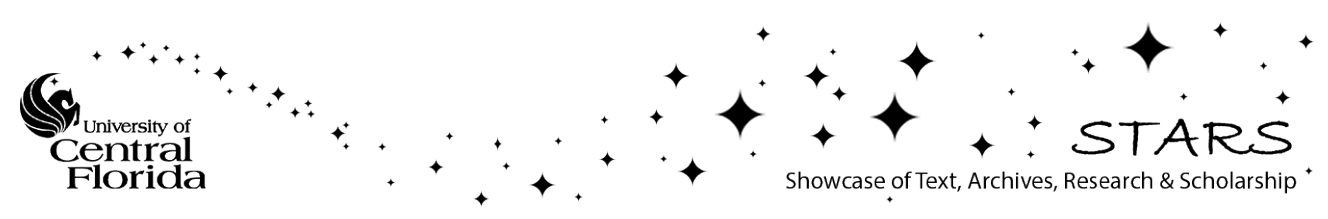




\section{Tunable Fresnel lens using nanoscale polymer-dispersed liquid crystals}

Cite as: Appl. Phys. Lett. 83, 1515 (2003); https://doi.org/10.1063/1.1604943

Submitted: 05 May 2003. Accepted: 27 June 2003. Published Online: 19 August 2003

Hongwen Ren, Yun-Hsing Fan, and Shin-Tson Wu

ARTICLES YOU MAY BE INTERESTED IN

Tunable-focus flat liquid crystal spherical lens

Applied Physics Letters 84, 4789 (2004); https://doi.org/10.1063/1.1760226

High-efficiency and economical solar-energy-pumped laser with Fresnel lens and chromium codoped laser medium

Applied Physics Letters 90, 261120 (2007); https://doi.org/10.1063/1.2753119

Tunable electronic lens using a gradient polymer network liquid crystal

Applied Physics Letters 82, 22 (2003); https://doi.org/10.1063/1.1534915

\section{Applied Physics Letters}

Mid-IR and THz frequency combs special collection

Read Now! 


\title{
Tunable Fresnel lens using nanoscale polymer-dispersed liquid crystals
}

\author{
Hongwen Ren, Yun-Hsing Fan, and Shin-Tson $\mathrm{Wu}^{\mathrm{a})}$ \\ School of Optics/CREOL, University of Central Florida, Orlando, Florida 32816
}

(Received 5 May 2003; accepted 27 June 2003)

\begin{abstract}
An electrically tunable Fresnel zone plate lens is demonstrated using nanoscale polymer-dispersed liquid crystal droplets. In the voltage-off state, the zone plate behaves like a positive lens. The measured diffraction efficiency is close to the theoretical limit and is controllable by voltage. The major advantages of such a Fresnel lens are simple fabrication, large aperture size, polarization independent, and fast switching speed, although the required operating voltage is relatively high. (C) 2003 American Institute of Physics. [DOI: 10.1063/1.1604943]
\end{abstract}

An electrically tunable liquid crystal (LC) lens is a critical component for optical information processing, optical interconnection, beam deflector, and three-dimensional (3D) display systems. Various LC lenses have been developed ${ }^{1-10}$ wherein the zone-plate Fresnel lens is most promising. In comparison with the refractive lenses, the Fresnel lens is simple to fabricate with large aperture size. Fresnel lenses are suitable for long distance optical communication, optical distance measurement, and space navigation. In a binaryphase planar Fresnel lens, the phase difference between the adjacent zones can be tuned by the applied voltage. Maximum diffraction occurs when the phase difference is equal to an odd multiple of $\pi$ while no diffraction occurs for an even multiple of $\pi$. Through diffraction, the Fresnel zone plate works as a focusing element.

Two techniques have been developed for fabricating LC Fresnel lens. One is to etch the indium-tin-oxide (ITO) electrode by electron beam lithography to form a zone plate ${ }^{3,5}$ and the other is to make the two neighboring zones with orthogonal LC directors. ${ }^{4}$ However, these fabrication methods are rather complicated. Recently, we have demonstrated a tunable electronic lens using a gradient refractive index nanoscale polymer-dispersed liquid crystal (GRIN PDLC). ${ }^{11}$ The key element for fabricating the GRIN PDLC lens is a patterned photomask with circularly variable optical density in the radical direction.

In this letter, we report the fabrication of a Fresnel lens using a photomask with zone plate patterns. The LC/ monomer in the zones cured with a higher UV intensity lead to smaller LC droplets. Conversely, the zones with a weaker UV exposure result in larger nanoscale droplets. When a uniform voltage is applied to the zone plate, the refractive index (or phase) of the zones with larger LC droplets are changed so that the beam diffraction efficiency and the focusing behaviors of the lens can be adjusted. Since the nanoscale LC droplets in the polymer matrix are randomly distributed, the lens is polarization independent and exhibits a fast switching time.

Figure 1 illustrates the fabrication method of a Fresnel lens using nanoscale PDLC. The key element is the photomask (right) which has transparent odd zones and opaque even zones. Our photomask was produced by etching a chro-

${ }^{a)}$ Electronic mail: swu@ mail.ucf.edu mium oxide layer using electron beam lithography. The radius $r_{1}$ of the innermost zone is $0.5 \mathrm{~mm}$ and the radius of the $n$th zone $\left(r_{n}\right)$ is given by $r_{n}^{2}=n r_{1}^{2}, n$ is the zone number. Our Fresnel zone plate consists of 80 zones in $1 \mathrm{~cm}$ aperture.

In our experiments, we mixed $26 \%$ nematic E48 LC $(\Delta n=0.231$ and $\Delta \varepsilon \sim 12.5)$ in an UV curable prepolymer NOA81 (Norland) host. Since NOA81 is sensitive to UV, the polymerization rate is relatively fast. The E48/NOA81 mixture was injected into a homogeneous LC cell composing of ITO-coated glass substrates. The cell gap is $d=15 \mu \mathrm{m}$. The bottom substrate surface is in proximity contact with the photomask. To avoid incomplete polymerization in the opaque zones, we illuminate the LC cell from both sides. A strong UV light $\left(I \sim 25 \mathrm{~mW} / \mathrm{cm}^{2}\right)$ illuminated from the bottom (photomask) and a weak UV light $\left(I \sim 10 \mathrm{~mW} / \mathrm{cm}^{2}\right)$ from the top of the cell for $20 \mathrm{~s}$. Thus, the odd zones experience a higher $\mathrm{UV}$ intensity $\left(I \sim 35 \mathrm{~mW} / \mathrm{cm}^{2}\right)$ than the even zones $\left(I \sim 10 \mathrm{~mW} / \mathrm{cm}^{2}\right)$ so that their droplet sizes are smaller. In both zones, the PDLC droplets are in subwavelength scale. Thus, they do not scatter visible light and their electro-optic properties are independent of the incident light polarization.

The odd zones of the prepared PDLC sample is highly transparent in the voltage-off state, while the even zones appear slightly bluish which implies the formed LC droplet is comparable to a blue wavelength. The Fresnel zone plate was inspected using a polarizing optical microscope where the sample was set between two crossed polarizers. At $V=0$, the birefringence colors of the Fresnel zones can be clearly observed (not shown). Such a Fresnel lens belongs to the binary-phase category. In a binary phase Fresnel lens, the

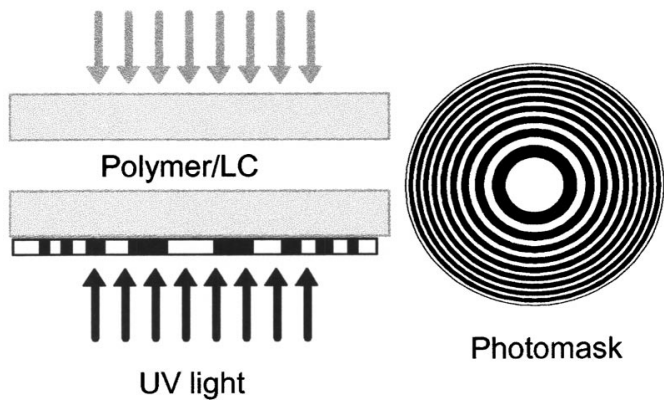

FIG. 1. Left: method for fabricating nano-PDLC Fresnel lens. Right: the photomask showing Fresnel zone structure. 


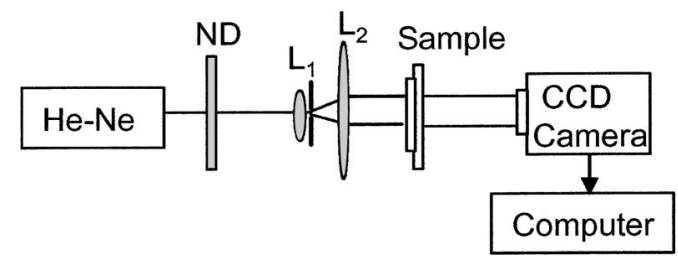

FIG. 2. Experimental setup for studying the PDLC Fresnel lens. ND $=$ neutral density filter, $L_{1}$ and $L_{2}$ are lenses, $\lambda=633 \mathrm{~nm}$.

focal length $f$ is related to the innermost zone radius $r_{1}$ as $f=r_{1}^{2} / \lambda$, where $\lambda$ is the wavelength of the incident beam. The primary focal length of our lens was $\sim 50 \mathrm{~cm}$ (for $\lambda$ $=633 \mathrm{~nm}$ ). Due to the higher-order Fourier components, Fresnel zone lens has multiple foci at $f, f / 3, f / 5$, etc. However, the majority of the incident light diffracts into the primary focus. The theoretical diffraction efficiency of the primary focus for the binary-phase Fresnel lens is $41 \% .^{12}$

To characterize the light focusing properties of the PDLC Fresnel lens, we measured the image quality and the $3 \mathrm{D}$ spot intensity profiles. Figure 2 depicts the experimental setup. The collimated $\mathrm{He}-\mathrm{Ne}$ laser beam has $\sim 1 \mathrm{~cm}$ diameter which fills the entire zone plate. To study the light focusing properties of the primary focal point, the charge coupled device (CCD) camera (SBIG model ST-2000XM) was set at $50 \mathrm{~cm}$ from the Fresnel lens and the data were analyzed by a computer.

To evaluate the image quality of the PDLC Fresnel lens, a black cardboard with transparent alphabet $R$ was placed between lens $L_{2}$ and sample. The CCD camera was set at $\sim 25 \mathrm{~cm}$ behind the sample. Figures 3(a) and 3(b) show the photos without and with the sample, respectively. When the sample is absent, no focusing effect occurs. Once the LC Fresnel lens is in position, a clear but smaller image is observed, although some circular noises exist due to diffraction. When the CCD camera was moved to the focal point, a tight spot appeared in the center, as shown in Fig. 3(c). These results indicate that the sample indeed behaves like a lens.

To characterize the voltage-dependent light diffraction efficiency, we measured the 3D profiles of the outgoing beams using a digital CCD camera. Results are shown in Fig. 4. In the absence of the LC sample, the collimated light intensity is relatively uniform and the average intensity is 300 arbitrary units, as shown in Fig. 4(a). When the nanoPDLC Fresnel zone plate is in position, a sharp focus occurs at $\sim 50 \mathrm{~cm}$. Figures 4(b) and 4(c) show the transmitted beam profiles at $V=0$ and $180 \mathrm{~V}_{\text {rms }}$, respectively. The peak intensity reaches $\sim 10^{4}$. As the driving voltage increases, the peak intensity gradually increases. At $V=180 \mathrm{~V}_{\mathrm{rms}}$, the peak in-

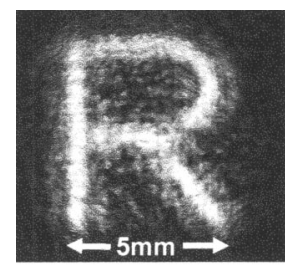

(a)

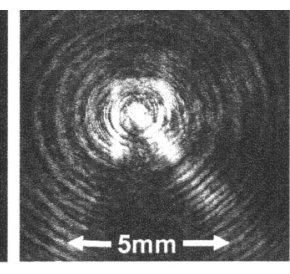

(b)

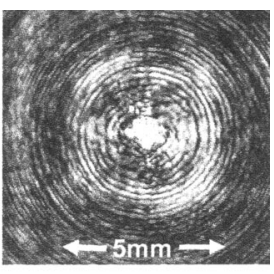

(c)
FIG. 3. Imaging and focusing properties of the PDLC Fresnel lens recorded by a CCD camera. (a) Without sample, (b) CCD at $25 \mathrm{~cm}$ from sample, and (c) $\mathrm{CCD}$ at focal point.

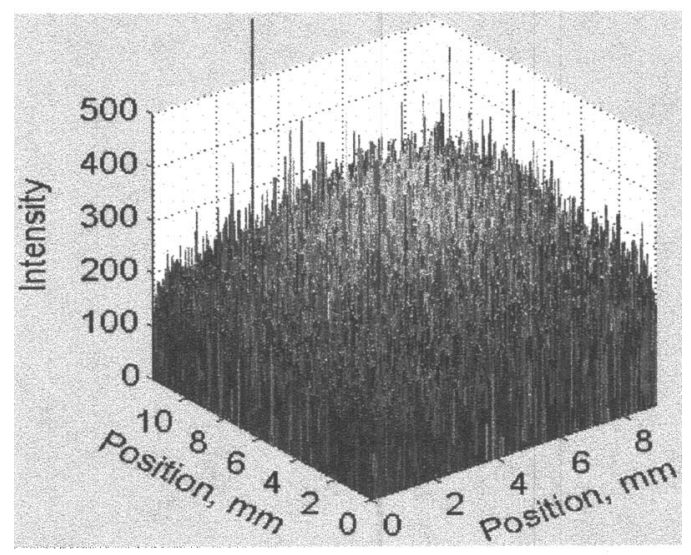

(a)

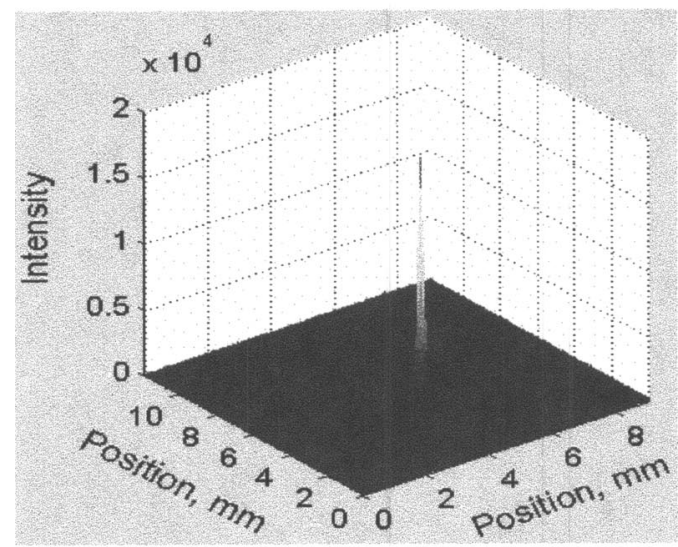

(b)

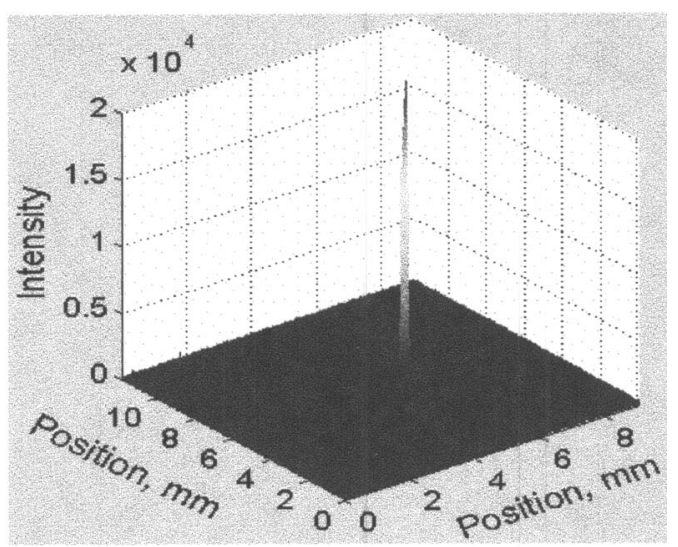

(c)

FIG. 4. Spot intensity profiles measured by the CCD camera under different conditions. (a) Without sample, (b) with sample at $V=0$, and (c) $V$ $=180 \mathrm{~V}_{\mathrm{rms}}$. The diameter of the Fresnel zone plate is $1 \mathrm{~cm}$ and the focal length is $50 \mathrm{~cm}$.

tensity is nearly $1.5 \mathrm{X}$ as compared to that at $V=0$. Further increasing voltage would reorient the droplets along the electric field and then decrease the light intensity in the focal spot. To avoid the electrode breakdown, our maximum voltage is limited to $225 \mathrm{~V}_{\text {rms }}$.

The diffraction efficiency $(\eta)$ of the Fresnel zone plate is defined as

$$
\eta=\frac{p-p_{0}}{p_{t}} .
$$




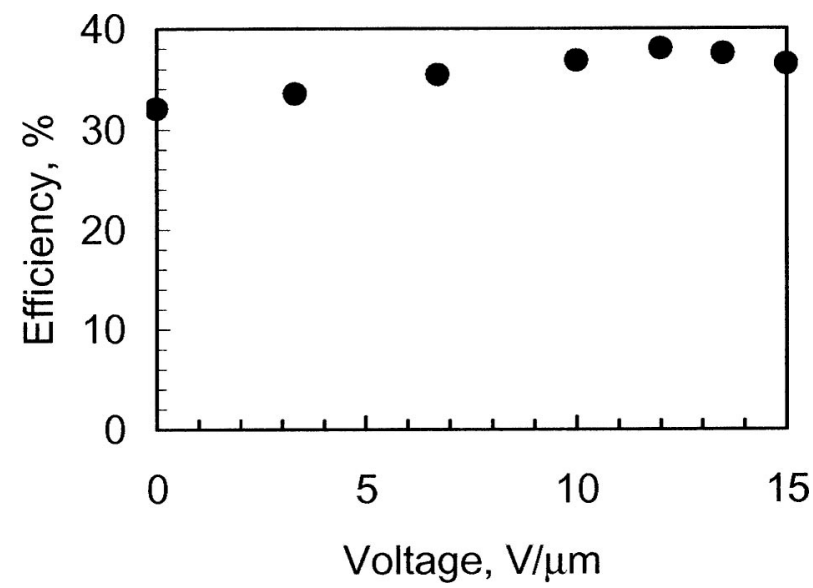

FIG. 5. Voltage-dependent diffraction efficiency of the PDLC Fresnel lens. The LC employed is E48, cell gap $d=15 \mu \mathrm{m}$, and $\lambda=633 \mathrm{~nm}$.

In Eq. (1), $P$ denotes the transmitted laser power at the focal point, $P_{0}$ is the background noise, and $P_{t}$ is the total incident power after passing through the sample. In this experiment, we used a photodetector to measure the diffracted light power. Results are plotted in Fig. 5. At $V=0$, the diffraction efficiency is $\eta \sim 32 \%$. As the voltage increases to $V$ $=180 \mathrm{~V}_{\mathrm{rms}}\left(\right.$ or $\left.12 \mathrm{~V}_{\mathrm{rms}} / \mu \mathrm{m}\right)$, the diffraction efficiency increases to $\sim 39 \%$, which is approaching the $41 \%$ theoretical limit. As the operating voltage exceeds $15 \mathrm{~V}_{\mathrm{rms}} / \mu \mathrm{m}, \eta$ starts to decline.

The first order diffraction efficiency of a Fresnel lens is determined by the relative phase difference $(\Delta \delta)$ between the adjacent zones

$$
\Delta \delta=2 \pi d\left(n_{\text {even }}-n_{\text {odd }}\right) / \lambda,
$$

where $d$ is the cell gap, and $n_{\text {even }}$ and $n_{\text {odd }}$ are the effective refractive index of the LC composite in even and odd zones, respectively. The maximum efficiency is reached when $\Delta \delta$ $\sim \pi$. Our results plotted in Fig. 5 imply that the initial $\Delta \delta$ at $V=0$ is slightly larger than $\pi$. As the applied voltage exceeds $\sim 3 \mathrm{~V} / \mu \mathrm{m}$, the LC composites in the even rings start to be reoriented by the electric field while the odd rings remain basically unaffected due to their smaller droplet sizes. Thus, $\Delta \delta$ is approaching $\pi$ and diffraction efficiency slightly increases. As the voltage keeps on increasing, the LC composites in the even and odd rings are both reoriented by the electric field. Beyond $12 \mathrm{~V} / \mu \mathrm{m}$, both $n_{\text {even }}$ and $n_{\text {odd }}$ are reduced and $\Delta \delta$ drops to below $\pi$. From this point on, the diffraction efficiency declines continuously.

The switching speed of the lens was measured using a square wave of $100 \mathrm{~ms}$ width and $180 \mathrm{~V}_{\mathrm{rms}}(1 \mathrm{kHz})$ pulse amplitude. The rise and decay times were measured to be $200 \mu$ s. From this result, we estimate that the average LC droplet size in the even zones is $\sim 300 \mathrm{~nm}$. The odd zones should have a smaller droplet size due to the stronger UV exposure.

Similar to other composite systems, ${ }^{13}$ our subwavelength LC composites would also have imperfect spherical droplet shapes and nonuniform size distribution. The required voltage to reorient these fine droplets is relatively high. Holographic PDLC is such an example. ${ }^{14}$ Ideally, if the LC droplets can be completely reoriented by the electric field and the ordinary refractive index of the LC matches that of the polymer matrix, then $\Delta \delta \rightarrow 0$ and $\eta \rightarrow 0$ would occur in the high voltage regime. However, it is difficult to make $n_{\text {odd }}=n_{\text {even }}$ because the smaller LC droplets in the odd zones experience a stronger anchoring force than those in the even zones. Thus, the required voltage for completely turning off the Fresnel diffraction would be very high.

To overcome the high voltage problem of the subwavelength PDLC Fresnel lens, several approaches can be considered. For example, one can use a thinner cell filled with a high birefringence $(\Delta n>0.4)$ and high $\Delta \varepsilon$ LC. Adding a small amount of surfactant to the PDLC film is found useful for lowering the voltage while retaining a high diffraction efficiency. ${ }^{15}$

In conclusion, we have demonstrated an electrically tunable Fresnel lens using a nano-PDLC zone plate. Such a lens is polarization independent and has fast switching speed. The diffraction efficiency of the Fresnel lens we fabricated approaches the theoretical limit at $\sim 12 \mathrm{~V} / \mu \mathrm{m}$.

The authors are indebted to Yi-Pai Huang for designing the photomask. This work is supported by DARPA under Contract No. DAAD19-02-1-0208.

${ }^{1}$ S. Sato, Jpn. J. Appl. Phys. 18, 1679 (1979).

${ }^{2}$ S. T. Kowel, P. Kornreich, and A. Nouhi, Appl. Opt. 23, 2774 (1984).

${ }^{3}$ G. Williams, N. J. Powell, A. Purvis, and M. G. Clark, Proc. SPIE 1168, 352 (1989).

${ }^{4}$ J. S. Patel and K. Rastani, Opt. Lett. 16, 532 (1991).

${ }^{5}$ H. Ren, L. Zhang, L. Xuan, M. Chen, and X. Huang, Soc. Info. Display Tech. Digest 32, 366 (2001).

${ }^{6}$ N. A. Riza and M. C. DeJule, Opt. Lett. 19, 1013 (1994).

${ }^{7}$ S. Masuda, S. Takahshi, T. Nose, S. Sato, and H. Ito, Appl. Opt. 36, 4772 (1997).

${ }^{8}$ W. W. Chan and S. T. Kowel, Appl. Opt. 36, 8958 (1997).

${ }^{9}$ A. F. Naumov and M. Y. Loktev, Opt. Lett. 23, 992 (1998).

${ }^{10}$ V. V. Presnyako, K. E. Asatryan, and T. V. Galstian, Opt. Express 10, 865 (2002).

${ }^{11}$ H. Ren and S. T. Wu, Appl. Phys. Lett. 81, 3537 (2002).

${ }^{12}$ T. Sakano, K. Naguchi, and T. Matsumoto, Appl. Opt. 29, 1094 (1990).

${ }^{13}$ S. Matsumoto, M. Houlbert, T. Hayashi, and K. Kubodera, Appl. Phys. Lett. 69, 1044 (1996).

${ }^{14}$ R. L. Sutherland, V. P. Tondiglia, L. V. Natarajan, T. J. Bunning, and W. W. Adams, Appl. Phys. Lett. 64, 1074 (1994).

${ }^{15}$ V. P. Tondiglia, L. V. Natarajan, R. L. Sutherland, T. J. Bunning, and W. W. Adams, Opt. Lett. 20, 1325 (1995). 